\title{
SURGICAL INJURY OF THE CORD AND ITS ROOTS
}

\author{
By Bernard J. Sussman, M.D. \\ Professor of Neurosurgery, Howard University College of Medicine, \\ Washington, D.C. 20059, U.S.A.
}

\section{Introduction}

Neurological deficit and pain are the anticipated consequence of disease which acts upon the vertebral column. That abnormal signs or symptoms may occur only as a complication of surgery upon the spine, or that existing symptoms may be aggravated rather than relieved by operation, has not gone unnoticed. Such clinical events are currently reported as a requirement during the course of a patient's hospitalisation and are subject to review. Innumerable case reports in the medical literature also attest to their prevalence and even entire books have been devoted to this subject (Horwitz \& Rizzoli, 1967). Almost invariably, however, these discussions narrow down to methods for avoidance, recognition of specific oversights, errors in technique or instrumentation, and recount the issue in terms of currently acceptable or non-acceptable percentages. Why surgeons may fall into error seems not to be an acceptable subject for discussion and to criticise those who do, at least from the scientific standpoint, would hardly occur to a medical writer. The net effect is that while aiming for better scores on these performances, surgeons still do tend to think of such complications rather as annoying intrusions, and much the same as others of us might regard the chance and quite inescapable flatness of a tyre.

It is also not unusual that when patients who are injured complain of their complications, surgeons are often inclined to write off their predicament as the inevitable consequence of an ever-advancing surgical frontier armed with more and more innovative, necessarily dangerous, and therefore potentially crippling techniques. Official representatives of such surgical specialists insist, without offering any supportive evidence that the major problem is 'not incompetent physicians' but rather 'poor' or 'adverse results of medical treatment' (Rovitt, 1976). Hardly a passing thought is directed toward the overall capability of every surgeon who is associated with such results. Nor does it seem that any effort is made in any particular circumstance to determine if more than one thing was out of hand when a specific problem arose. The most that one occasionally hears is the grudging admission that there probably do exist a very limited number of incompetent surgeons who could account for a few unfortunate incidents which may be avoidable and that some housecleaning is in order by better peer review. But about this there are vagaries of pronouncement, commitment and fulfilment. The conventional insistence goes on to claim that patients may be presumed to be otherwise safe in the hands of all the other surgeons who are now required to pursue a continuing post-graduate educational experience.

In my own specialty, that of neurosurgery, the only official concessions are that we are 'surely not entirely blameless' for 'neurosurgery has advanced rapidly and it is no great surprise that all neurosurgeons are not equally competent especially in all varieties of the specialty' (Rovitt, 1976). The relevance of such 
pronouncements must yield to the fact that in the series of cases I now analyse and report, we are confronted solely by complications related to commonplace conditions requiring treatment in an ordinary manner and by very basic surgical skills which the surgeon is supposed to master as a beginner in his training, much less in his subsequent practice. In fact, the question has never been raised with me of the propriety of treatment in any circumstance related to a new technique of great risk and requiring unusual skills. One should also remember that even such methods must satisfy the requirement of a favourable benefit to risk ratio and must meet the test of reasonable safety.

Such tenets of my profession, however, are herewith further tested in a detailed review of 52 complications related to the surgical treatment of disorders of the spine.

\section{Clinical Data and Statistics}

The hospital charts and the radiographs of 52 patients were studied. When available, the records and the radiographs of subsequent hospitalisations or outpatient examinations were also examined. The reports and statements of treating or consulting doctors were read.

These clinical records are summarised in Tables IA, B, C and D.

All operations involving the cervical spine (20) were performed by neurological surgeons. Of these there were I3 posterior laminectomies and seven anterior interbody explorations usually with fusion.

Of 12 original thoracic or thoraco-lumbar procedures, ten were carried out by neurological surgeons. Two patients were operated upon at these levels primarily or secondarily by orthopaedic surgeons. There were nine posterior laminectomies, one lateral operation for disc, one multiple osteotomy for scoliosis done orthopaedically and one spinal cord angiogram for cervical osteo-arthritis by a radiologist.

Sixteen lumbar procedures for herniated disc were evenly divided between neurological and orthopaedic surgeons. The only child in this study was operated upon by a general surgeon for a lumbar 'hemangioma'.

One lumbar and one thoracic laminectomy to relieve pain by rhizotomy as well as one lumbar intrathecal injection of hypertonic saline were carried out by neurological surgeons.

Sex. Forty patients were male and 12 were female.

Conservative treatment. In 40 clinical circumstances where such treatment was indicated the needs of the patient were not met in 36. This represents a failure rate of 90 per cent.

Diagnostic techniques. These were generally suboptimal but nevertheless they were sufficient in 33 of 48 patients (69 per cent) to permit accurate diagnosis.

Error in diagnosis. This occurred, in spite of the foregoing observation, in $3 \mathrm{I}$ of 48 patients (65 per cent). Two additional diagnostic errors were on clinical grounds.

Surgical technique. In 50 circumstances where the issue of surgical adequacy was relevant, surgery failed to meet accepted standards in 43 . This constitutes an 86 per cent failure rate. A general tabulation of deviations, in chronological order, is included in Table II. Surgical deviations which occurred during anterior cervical procedures are listed separately in Table III. It would not appear that those operators who elected to do the anterior cervical interbody procedure were generally familiar to a sufficient degree with the necessary instrumentation and 
TABLE IA

Cervical

\begin{tabular}{|c|c|c|c|c|c|c|c|c|c|c|}
\hline & Sex & $\begin{array}{l}\text { Neuro- } \\
\text { surgeon }\end{array}$ & $\begin{array}{l}\text { Ortho- } \\
\text { paedic } \\
\text { surgeon }\end{array}$ & $\begin{array}{l}\text { Conser- } \\
\text { vative } \\
\text { treatment }\end{array}$ & $\begin{array}{l}\text { Diagnostic } \\
\text { technique }\end{array}$ & Diagnosis & $\begin{array}{l}\text { Appropriate } \\
\text { diagnosis }\end{array}$ & $\begin{array}{l}\text { Surgical } \\
\text { technique }\end{array}$ & $\begin{array}{l}\text { Post- } \\
\text { operative } \\
\text { care }\end{array}$ & Course \\
\hline I & $M$ & + & & - & - & $\begin{array}{l}\text { Herniated disc } \\
\text { C5-6 and } \\
\text { C6-7 }\end{array}$ & $\begin{array}{l}\text { Spondylotic } \\
\text { radiculopathy } \\
\text { C5-C8 left }\end{array}$ & - & - & $\begin{array}{l}\text { Anterior fusion } 2 \text { levels. Severe weakness } \\
\text { both upper limbs and right lower limb, } \\
\text { sensory level, neurogenic bladder. }\end{array}$ \\
\hline 2 & $M$ & + & & - & + & $\begin{array}{l}\text { Herniated disc } \\
\text { C5-6 and C6-7 }\end{array}$ & $\begin{array}{l}\text { Spondylotic } \\
\text { radiculopathy } \\
\text { C5-6 }\end{array}$ & - & - & $\begin{array}{l}\text { Anterior fusion } 2 \text { levels. Paraparesis after } \\
\text { post-operatuve quadriplegia from haema- } \\
\text { toma. }\end{array}$ \\
\hline 3 & $M$ & + & & - & + & $\begin{array}{l}\text { Herniated disc } \\
\text { C6-7 }\end{array}$ & $\begin{array}{l}\text { Spondylotic } \\
\text { radiculopathy } \\
\mathrm{C}_{5}-\mathrm{C}_{7}\end{array}$ & - & - & $\begin{array}{l}\text { Anterior fusion C6-7 with graft in spinal } \\
\text { canal. Quadriplegia. }\end{array}$ \\
\hline 4 & $M$ & + & & - & - & $\begin{array}{l}\text { Herniated disc } \\
\text { C6-7 }\end{array}$ & $\begin{array}{l}\text { Spondylotic } \\
\text { radiculopathy } \\
\text { C6-7 }\end{array}$ & - & - & $\begin{array}{l}\text { Anterior fusion C6-7. Hemiparesis, } \\
\text { bilateral sensory level. }\end{array}$ \\
\hline 5 & $M$ & + & & - & + & $\begin{array}{l}\text { Degenerated disc } \\
\text { C4-5 }\end{array}$ & $\begin{array}{l}\text { Spondylotic } \\
\text { radiculopathy } \\
\text { C4-C8 }\end{array}$ & - & - & $\begin{array}{l}\text { Anterior fusion } \mathrm{C}_{4}-5 \text { with graft in spinal } \\
\text { canal. Quadriplegia. }\end{array}$ \\
\hline 6 & $\mathrm{~F}$ & + & & - & - & $\begin{array}{l}\text { Herniated disc. } \\
\text { Cervical rib }\end{array}$ & $\begin{array}{l}\text { Cervical root } \\
\text { contusion }\end{array}$ & - & - & $\begin{array}{l}\text { Anterior fusion } \mathrm{C}_{3}-4, \mathrm{C}_{4}-5, \mathrm{C}_{5}-6, \mathrm{C} 6-7 . \\
\text { Four operations for cervical rib. Sympa- } \\
\text { thectomy. Stiff neck, weakness and } \\
\text { causalgia upper limb. }\end{array}$ \\
\hline 7 & $M$ & + & & - & + & $\begin{array}{l}\text { Herniated disc } \\
\text { C6-7 }\end{array}$ & $\begin{array}{l}\text { Angina. Spondy- } \\
\text { lotic radiculo- } \\
\text { pathy } \mathrm{C}_{5}-6\end{array}$ & - & - & $\begin{array}{l}\text { Anterior fusion } \mathrm{C}_{5}-6 \text { and C6-7. Interrup- } \\
\text { tion vertebral artery. Paralysis opposite } \\
\text { upper limb. Homonymous hemianopsia. } \\
\text { Root injury. }\end{array}$ \\
\hline 8 & $M$ & + & & - & - & Myelopathy $\mathrm{C}_{5}$ & $\begin{array}{l}\text { Radiculopathy } \\
\text { C4-5 }\end{array}$ & + & + & $\begin{array}{l}\text { Prophylactic decompressive laminectomy. } \\
\text { Upper limbs paralysed and sensory loss by } \\
\text { severe central cord injury. }\end{array}$ \\
\hline 9 & $M$ & + & & $\ldots$ & - & $\begin{array}{l}\text { Spinal cord } \\
\text { tumor } \mathrm{C}_{5}-6\end{array}$ & $\begin{array}{l}\text { Cervical disc } \\
\text { C5-6 }\end{array}$ & - & - & $\begin{array}{l}\text { Limited laminectomy. Anterior fusion. } \\
\text { From partial Brown-Sequard to ascending } \\
\text { quadriplegia and death. }\end{array}$ \\
\hline Io & $M$ & + & & - & + & $\begin{array}{l}\text { Spondylosis with } \\
\text { C6-7 partial block } \\
\text { right }\end{array}$ & $\begin{array}{l}\text { Spondylosis } \\
\text { bilateral } \mathrm{C}_{4}-\mathrm{C}_{7} \\
\text { left }>\text { right }\end{array}$ & - & - & $\begin{array}{l}\text { Longitudinal laminectomy } \mathrm{C}_{3}-\mathrm{C}_{7} \text { right }> \\
\text { left. Radiculopathy to quadriplegia from } \\
\text { post-operative clot. Residual atrophy } \\
\text { upper limbs, neurogenic bladder, pyra- } \\
\text { midal and sensory deficits. }\end{array}$ \\
\hline I I & $M$ & + & & $\ldots$ & + & $\begin{array}{l}\text { Spondylotic } \\
\text { Myelopathy }\end{array}$ & $\begin{array}{l}\text { Amyotrophic } \\
\text { lateral sclerosis }\end{array}$ & + & + & $\begin{array}{l}\text { Longitudinal laminectomy. Post- } \\
\text { operative hematoma. Quadriplegia. }\end{array}$ \\
\hline
\end{tabular}




\begin{tabular}{|c|c|c|c|c|c|c|c|c|c|}
\hline 12 & M & + & + & + & $\begin{array}{l}\text { Spondylotic } \\
\text { Myelopathy }\end{array}$ & $\begin{array}{l}\text { Spondylotic } \\
\text { Myelopathy }\end{array}$ & - & - & $\begin{array}{l}\text { Longitudinal laminectomy. From mild } \\
\text { Brown-Sequard to pseudobulbar quadri- }\end{array}$ \\
\hline I3 & M & + & - & + & $\begin{array}{l}\text { Spondylotic } \\
\text { radiculopathy } \\
\text { bilateral }\end{array}$ & $\begin{array}{l}\text { Spondylotic } \\
\text { radiculopathy } \\
\text { C5-6, C6-7 left }\end{array}$ & - & - & $\begin{array}{l}\text { Bilateral foramenotomies with drill C5-6 } \\
\text { and C6-7. Longitudinal laminectomy } \\
\text { C4-7. Dentates cut. Dura left open. } \\
\text { Contralateral upper monoparesis, para- } \\
\text { paresis, neurogenic bladder, sensory level } \\
\text { and persistent pain. }\end{array}$ \\
\hline I 4 & $M$ & + & - & + & $\begin{array}{l}\text { Herniated disc } \\
\mathrm{C}_{5}-6\end{array}$ & $\begin{array}{l}\text { Spondylotic } \\
\text { radiculopathy } \\
\text { C5-6 }\end{array}$ & - & - & $\begin{array}{l}\text { Limited laminectomy and foramenotomy. } \\
\text { Severe upper monoparesis. Brown- } \\
\text { Sequard with paraparesis. }\end{array}$ \\
\hline I 5 & $M$ & + & - & + & $\begin{array}{l}\text { Spondylotic } \\
\text { radiculopathy } \\
\text { or Herniated } \\
\text { disc C5-6 }\end{array}$ & $\begin{array}{l}\text { Spondylotic } \\
\text { radiculopathy } \\
\text { C5-6 }\end{array}$ & - & - & $\begin{array}{l}\text { Limited laminectomy and retained cot- } \\
\text { tonoid sponge. Upper monoparesis and } \\
\text { causalgic cervical pain. }\end{array}$ \\
\hline I6 & $M$ & + & + & + & $\begin{array}{l}\text { Cord compres- } \\
\text { sion } \mathrm{C}_{7}-\mathrm{T}_{\mathrm{I}}\end{array}$ & $\begin{array}{l}\text { Cord compres- } \\
\text { sion } C_{7}-T_{I}\end{array}$ & - & - & $\begin{array}{l}\text { Longitudinal laminectomy. Paraplegia } \\
\text { after placed in chair first post-operation } \\
\text { day. }\end{array}$ \\
\hline 17 & $M$ & + & + & + & $\begin{array}{l}\text { Spondylotic } \\
\text { radiculopathy } \\
\text { C6-7 }\end{array}$ & $\begin{array}{l}\text { Spondylotic } \\
\text { radiculopathy } \\
\text { C6-7 }\end{array}$ & - & - & $\begin{array}{l}\text { Limited laminectomy. Dura left open over } \\
\text { root. Paraparesis from arachnoid cyst. }\end{array}$ \\
\hline I 8 & $\mathrm{~F}$ & + & - & + & $\begin{array}{l}\text { Neurofibroma } \\
\mathrm{C}_{4}-5\end{array}$ & $\begin{array}{l}\text { Spondylotic } \\
\text { radiculopathy } \\
\text { C4-6. Spinal } \\
\text { stenosis }\end{array}$ & - & - & Limited laminectomy. Quadriplegia. \\
\hline I9 & M & + & $\cdots$ & + & $\begin{array}{l}\text { Cord compres- } \\
\text { sion } \mathrm{C}_{7}-\mathrm{T}_{4} \\
\text { Pagets }\end{array}$ & $\begin{array}{l}\text { Cord compres- } \\
\text { sion T8-9 Pagets }\end{array}$ & - & - & $\begin{array}{l}\text { Longitudinal laminectomy upper level. } \\
\text { Paraplegia. }\end{array}$ \\
\hline 20 & M & + & - & + & $\begin{array}{l}\text { Cervical cord } \\
\text { compression } \\
\text { Haematomyelia } \\
\text { C3-4 }\end{array}$ & $\begin{array}{l}\text { Central cord } \\
\text { contusion } \mathrm{C}_{4}-7 . \\
\text { Herniated disc } \\
\mathrm{C}_{3-4}\end{array}$ & - & - & $\begin{array}{l}\text { Limited laminectomy. Incision spinal } \\
\text { cord. Dentates cut. Quadriplegia. }\end{array}$ \\
\hline
\end{tabular}

Brown-Sequard to pseudobulbar quadriplegia and impaired eye movements.

Bilateral foramenotomies with drill $\mathrm{C}_{5-6}$ 4-7. Dentates cut. Dura left open. Contralateral upper monoparesis, paraand persistent pain. 
TABLE IB

Thoracic and Thoracolumbar

\begin{tabular}{|c|c|c|c|c|c|c|c|c|c|c|}
\hline & Sex & $\begin{array}{l}\text { Neuro- } \\
\text { surgeon }\end{array}$ & $\begin{array}{l}\text { Ortho- } \\
\text { paedic } \\
\text { surgeon }\end{array}$ & $\begin{array}{l}\text { Conser- } \\
\text { vative } \\
\text { treatment }\end{array}$ & $\begin{array}{c}\text { Diagnostic } \\
\text { technique }\end{array}$ & Diagnosis & $\begin{array}{l}\text { Appropriate } \\
\text { diagnosis }\end{array}$ & $\begin{array}{l}\text { Surgical } \\
\text { technique }\end{array}$ & $\begin{array}{l}\text { Post- } \\
\text { operative } \\
\text { care }\end{array}$ & Course \\
\hline $2 I$ & $M$ & + & + & - & - & $\begin{array}{l}\text { Herniated disc } \\
\mathrm{T}_{4-5}\end{array}$ & $\begin{array}{l}\text { Herniated disc } \\
\mathrm{T}_{4-5}\end{array}$ & + & - & $\begin{array}{l}\text { Lateral approach. Paraplegia. 6-month } \\
\text { delay in spite of } 3 \text { abnormal myelograms } \\
\text { and high CSF protein. }\end{array}$ \\
\hline 22 & $M$ & + & & - & + & $\begin{array}{l}\text { Spinal cord } \\
\text { tumour T9-Io }\end{array}$ & $\begin{array}{l}\text { Herniated disc } \\
\text { T9-10 }\end{array}$ & - & - & $\begin{array}{l}\text { Laminectomy. Paraplegia. Extrusion } \\
\text { and biopsy of cord. Pre-operation } \\
\text { symptoms only radicular. }\end{array}$ \\
\hline 23 & $M$ & + & & $\ldots$ & - & $\begin{array}{l}\text { Cord compres- } \\
\text { sion T6-7 }\end{array}$ & $\begin{array}{l}\text { Extramedullary } \\
\text { tumour T4-6 }\end{array}$ & - & - & $\begin{array}{l}\text { Two laminectomies at inappropriate levels. } \\
\text { Progression from partial to complete para- } \\
\text { plegia. }\end{array}$ \\
\hline 24 & $\mathrm{~F}$ & + & & $\cdots$ & + & $\begin{array}{l}\text { Recurrent } \mathrm{T} 8 \\
\text { neurofibroma } \\
\text { after } 20 \text { years }\end{array}$ & Arachnoiditis & + & + & $\begin{array}{l}\text { Laminectomy. Progression from minimal } \\
\text { pyramidal signs to paraplegia. CSF pro- } \\
\text { tein normal and no pre-operation mass } \\
\text { lesion after } 20 \text { years. }\end{array}$ \\
\hline 25 & $\mathrm{~F}$ & + & & - & + & $\begin{array}{l}\text { Extradural } \\
\text { tumour T7-I I }\end{array}$ & $\begin{array}{l}\text { Arachnoiditis } \\
\text { T7-I I }\end{array}$ & - & - & $\begin{array}{l}\text { Laminectomy. Expired. No significant } \\
\text { pre-operative neurological deficit. }\end{array}$ \\
\hline 26 & $M$ & + & & $\ldots$ & + & $\begin{array}{l}\text { Epidural abscess } \\
\text { T8-9 }\end{array}$ & $\begin{array}{l}\text { Epidural abscess } \\
\text { T8-9 }\end{array}$ & - & - & $\begin{array}{l}\text { Laminectomy inappropriate level and } 2 \\
\text { level intradural posterior rhizotomies Tro- } \\
\text { TII. Paraparesis to paraplegia. }\end{array}$ \\
\hline 27 & $M$ & + & & $\cdots$ & + & $\begin{array}{l}\text { Hematomyelia } \\
\text { TI2 }\end{array}$ & $\begin{array}{l}\text { Hematomyelia } \\
\text { TI2 }\end{array}$ & - & - & $\begin{array}{l}\text { Laminectomy. Failure to detect or treat } \\
\text { low prothrombin. Paraplegia. }\end{array}$ \\
\hline 28 & $M$ & + & + & - & + & Scoliosis & Scoliosis & - & - & $\begin{array}{l}\text { Multiple osteotomies for cosmetic correc- } \\
\text { tion. Paraplegia after excessive bleeding } \\
\text { and delayed re-operation for haematoma. }\end{array}$ \\
\hline 29 & $\mathrm{~F}$ & & & $\ldots$ & - & $\begin{array}{l}\text { Cervical Spondy- } \\
\text { lotic Myelopathy }\end{array}$ & $\begin{array}{l}\text { Cervical Spondy- } \\
\text { lotic Myelopathy }\end{array}$ & $\ldots$ & - & $\begin{array}{l}\text { Paraplegia following thoracic spinal cord } \\
\text { angiogram by radiologist and requested by } \\
\text { neurosurgeon. }\end{array}$ \\
\hline 30 & $M$ & + & & $\ldots$ & - & Head injury & $\begin{array}{l}\text { Cord compres- } \\
\text { sion and fracture } \\
\mathrm{L}_{\mathrm{I}}\end{array}$ & + & + & $\begin{array}{l}\text { Laminectomy. Paraparesis to paraplegia } \\
\text { during 4-day delay to evacuation of } \\
\text { haematoma. }\end{array}$ \\
\hline $3 I$ & $M$ & + & & $\ldots$ & - & $\begin{array}{l}\text { Cauda equina } \\
\text { syndrome }\end{array}$ & $\begin{array}{l}\text { Cord compres- } \\
\text { sion TII-LI }\end{array}$ & - & - & $\begin{array}{l}\text { Limited laminectomy. No decompression } \\
\text { fracture Tir. Paraplegia after incomplete } \\
\text { cord injury. Delayed surgery with } \\
\text { myelographic block. }\end{array}$ \\
\hline 32 & $\mathrm{~F}$ & + & + & $\ldots$ & + & $\begin{array}{l}\text { Compression } \\
\text { fracture, Ti2 } \\
\text { block }\end{array}$ & $\begin{array}{l}\text { Compression } \\
\text { fracture, T I } 2 \\
\text { block }\end{array}$ & - & - & $\begin{array}{l}\text { Laminectomy done at } \mathrm{L} 2-3 \text { and then at } \\
\text { Tro-I I with biopsy of cord by orthopedic } \\
\text { surgeon. Paraplegia after incomplete cord } \\
\text { injury. }\end{array}$ \\
\hline
\end{tabular}

$\ldots=$ Not relevant. 
TABLE IC

Lumbar

\begin{tabular}{|c|c|c|c|c|c|c|c|c|c|c|}
\hline & Sex & $\begin{array}{l}\text { Neuro- } \\
\text { surgeon }\end{array}$ & $\begin{array}{l}\text { Ortho- } \\
\text { paedic } \\
\text { surgeon }\end{array}$ & $\begin{array}{l}\text { Conser- } \\
\text { vative } \\
\text { treatment }\end{array}$ & $\begin{array}{c}\text { Diagnostic } \\
\text { technique }\end{array}$ & Diagnosis & $\begin{array}{l}\text { Appropriate } \\
\text { diagnosis }\end{array}$ & $\begin{array}{l}\text { Surgical } \\
\text { technique }\end{array}$ & $\begin{array}{l}\text { Post- } \\
\text { operative } \\
\text { care }\end{array}$ & Course \\
\hline 33 & $M$ & & + & - & + & $\begin{array}{l}\text { Herniated disc } \\
\text { L5 }_{5} \text { I }\end{array}$ & 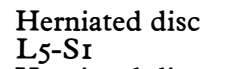 & - & + & $\begin{array}{l}\text { Severe pain increased by inadequate } \\
\text { removal. }\end{array}$ \\
\hline 34 & $\mathrm{~F}$ & & + & - & - & 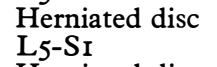 & $\begin{array}{l}\text { Herniated disc } \\
\text { L4-5. }\end{array}$ & - & - & $\begin{array}{l}\text { Extensive laminectomy. Weakness, } \\
\text { sensory loss, generalised pain, lower limbs. }\end{array}$ \\
\hline 35 & $M$ & & + & - & + & $\begin{array}{l}\text { Herniated disc } \\
\mathrm{L}_{3}-4\end{array}$ & 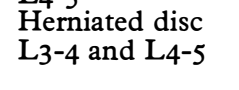 & - & - & $\begin{array}{l}\text { Operation at L2-3 and L } 3-4 \text {. Psoas and } \\
\text { caudal weakness, foot drop, pain, sensory } \\
\text { loss opposite lower limb. }\end{array}$ \\
\hline 36 & $M$ & + & & - & + & $\begin{array}{l}\text { Herniated disc } \\
{\text { L } 5-S_{I}}\end{array}$ & $\begin{array}{l}\text { Herniated disc } \\
\text { L5-SI }\end{array}$ & - & - & $\begin{array}{l}3 \text { operations including rhizotomy. } \\
\text { Paralysis and hypalgesia leg. Persistent } \\
\text { pain. }\end{array}$ \\
\hline 37 & $\mathrm{~F}$ & & + & - & - & $\begin{array}{l}\text { Herniated disc } \\
\text { L4-5 }\end{array}$ & $\begin{array}{l}\text { Herniated disc } \\
\mathrm{L}_{4}-5 \text { and } \mathrm{L}_{5}-\mathrm{S}_{\mathrm{I}}\end{array}$ & - & - & $\begin{array}{l}\text { Operation at one level with dural tear. } \\
\text { Persistent pain, weakness leg with sensory } \\
\text { loss. }\end{array}$ \\
\hline 38 & $M$ & + & & - & + & $\begin{array}{l}\text { Herniated disc } \\
\text { L4-5 }\end{array}$ & $\begin{array}{l}\text { Herniated disc } \\
\text { L4-5 }\end{array}$ & + & - & $\begin{array}{l}\text { Severe wound infection I year. Persistent } \\
\text { pain. }\end{array}$ \\
\hline 39 & $M$ & + & & - & + & $\begin{array}{l}\text { Tumour L4-5. } \\
\text { Herniated disc } \\
\text { L } 5-S I^{-}\end{array}$ & 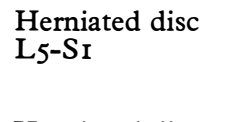 & - & - & $\begin{array}{l}\text { Laminectomy and intradural operation L4. } \\
\text { Neurogenic bowel and bladder, saddle } \\
\text { anaesthesia and pain from root injuries } \\
\text { and adhesive arachnoiditis. }\end{array}$ \\
\hline 40 & $M$ & & + & - & - & 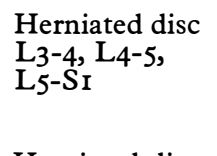 & $\begin{array}{l}\text { Herniated disc } \\
\text { L4-5 }\end{array}$ & - & - & $\begin{array}{l}\text { Operation at } 3 \text { levels. Atrophy, pain, } \\
\text { numbness lower limbs, neurogenic bladder } \\
\text { and bowel. Needle artefact L } 3-4, \text { L }_{5}-\mathrm{S}_{\mathrm{I}} \\
\text { defect opposite side. Dural tear and } \\
\text { arachnoiditis. }\end{array}$ \\
\hline $4 \mathrm{I}$ & $\mathrm{F}$ & + & & - & + & $\begin{array}{l}\text { Herniated disc } \\
\mathrm{L}_{4}-5, \mathrm{~L}_{5}-\mathrm{S}_{\mathbf{I}}\end{array}$ & $\begin{array}{l}\text { Herniated disc } \\
\mathrm{L}_{4}-5, \mathrm{~L}_{5}-\mathrm{SI}_{\mathrm{I}}\end{array}$ & - & - & $\begin{array}{l}\text { Seven operations. Persistent pain. } \\
\text { Arachnoiditis. }\end{array}$ \\
\hline 42 & $M$ & & + & - & + & $\begin{array}{l}\text { Herniated disc } \\
\text { L } 4-5 \text {. }\end{array}$ & $\begin{array}{l}\text { Herniated disc } \\
\text { L4-5 }\end{array}$ & - & - & $\begin{array}{l}\text { Laminectomy. Root torn, Paralysis leg, } \\
\text { saddle anaesthesia, neurogenic bladder. }\end{array}$ \\
\hline 43 & $\mathrm{~F}$ & + & & - & + & $\begin{array}{l}\text { Herniated disc } \\
\text { L4-5 }\end{array}$ & $\begin{array}{l}\text { Normal myelo- } \\
\text { gram }\end{array}$ & - & + & $\begin{array}{l}5 \text { operations. Rhizotomy. Severe pain, } \\
\text { weakness, numbness, lower limb. } \\
\text { Arachnoiditis. }\end{array}$ \\
\hline
\end{tabular}


Table Ic (continued)

\begin{tabular}{|c|c|c|c|c|c|c|c|c|c|c|}
\hline & Sex & $\begin{array}{l}\text { Neuro- } \\
\text { surgeon }\end{array}$ & $\begin{array}{l}\text { Ortho- } \\
\text { paedic } \\
\text { surgeon }\end{array}$ & $\begin{array}{l}\text { Conser- } \\
\text { vative } \\
\text { treatment }\end{array}$ & $\begin{array}{l}\text { Diagnostic } \\
\text { technique }\end{array}$ & Diagnosis & $\begin{array}{l}\text { Appropriate } \\
\text { diagnosis }\end{array}$ & $\begin{array}{c}\text { Surgical } \\
\text { technique }\end{array}$ & $\begin{array}{l}\text { Post- } \\
\text { operative } \\
\text { care }\end{array}$ & Course \\
\hline 44 & $M$ & & + & - & + & $\begin{array}{l}\text { Herniated disc } \\
\text { central and left } \\
\text { L4-5 }\end{array}$ & $\begin{array}{l}\text { Herniated disc } \\
\text { L4-5 left }\end{array}$ & - & - & $\begin{array}{l}\text { Laminectomy. Electro-coagulation. } \\
\text { Bilateral operation. Wasting, numbness } \\
\text { both legs. Neurogenic bowel and } \\
\text { bladder. }\end{array}$ \\
\hline 45 & $M$ & + & & - & + & $\begin{array}{l}\text { Herniated disc } \\
\text { L4-5 and } L_{5}-S_{1} \\
\text { left }\end{array}$ & $\begin{array}{l}\text { Herniated disc } \\
\text { L5-SI right }\end{array}$ & - & - & $\begin{array}{l}3 \text { operations and } 3 \text { myelograms. Progres- } \\
\text { sive paraplegia from root injury and } \\
\text { arachnoiditis. }\end{array}$ \\
\hline 46 & $M$ & & + & - & + & $\begin{array}{l}\text { Herniated disc } \\
\text { L4-5 }\end{array}$ & $\begin{array}{l}\text { Herniated disc } \\
\text { L4-5 }\end{array}$ & - & - & $\begin{array}{l}\text { Laminectomy. Paraparesis and neuro- } \\
\text { genic bladder. Delayed re-operation for }\end{array}$ \\
\hline 47 & $\mathrm{~F}$ & + & & + & - & $\begin{array}{l}\text { Herniated disc } \\
\text { L4-5 }\end{array}$ & $\begin{array}{l}\text { Herniated disc } \\
\text { L4-5 }\end{array}$ & - & - & $\begin{array}{l}\text { Operation } \mathrm{L}_{3}-4 . \text { Re-operation } \mathrm{L}_{4}-5 \text {. } \\
\text { Death from CNS infection after } 4 \text { attempts } \\
\text { to repair CSF fistula and discharged with } \\
\text { active drainage. }\end{array}$ \\
\hline 48 & $M$ & $*$ & & $\cdots$ & - & $\begin{array}{l}\text { Hemangioma } \\
\text { buttock }\end{array}$ & $\begin{array}{l}\text { Meningomyelo- } \\
\text { cele }\end{array}$ & - & - & $\begin{array}{l}\text { Transection neural tissue. Paraparesis. } \\
\text { Neurogenic bladder. }\end{array}$ \\
\hline 49 & $M$ & + & & - & + & $\ldots$ & $\ldots$ & $\cdots$ & $\cdots$ & $\begin{array}{l}\text { Myelogram for backache. Arachnoiditis. } \\
\text { Permanent abducent palsy and diplopia. }\end{array}$ \\
\hline
\end{tabular}

* Surgeon.

$\ldots=$ Not relevant. 
TABLE ID

Pain

\begin{tabular}{|c|c|c|c|c|c|c|c|c|c|c|}
\hline & Sex & $\begin{array}{l}\text { Neuro- } \\
\text { surgeon }\end{array}$ & $\begin{array}{l}\text { Ortho- } \\
\text { paedic } \\
\text { surgeon }\end{array}$ & $\begin{array}{l}\text { Conser- } \\
\text { vative } \\
\text { treatment }\end{array}$ & $\begin{array}{l}\text { Diagnostic } \\
\text { technique }\end{array}$ & Diagnosis & $\begin{array}{l}\text { Appropriate } \\
\text { diagnosis }\end{array}$ & $\begin{array}{l}\text { Surgical } \\
\text { technique }\end{array}$ & $\begin{array}{l}\text { Post- } \\
\text { operative } \\
\text { care }\end{array}$ & Course \\
\hline 50 & $\mathrm{M}$ & + & & - & - & $\begin{array}{l}\text { Intercostal } \\
\text { neuralgia } T_{5-9}\end{array}$ & $\begin{array}{l}\text { Hiatal hernia. } \\
\text { Post-operative } \\
\text { pain }\end{array}$ & - & - & $\begin{array}{l}\text { Laminectomy. Unilateral posterior } \\
\text { rhizotomy T } 4-9 \text {, paraparesis, sensory loss, } \\
\text { neurogenic bowel and bladder. }\end{array}$ \\
\hline $5 I$ & $\mathrm{~F}$ & + & & - & - & $\begin{array}{l}\text { Pain due to } \\
\text { fracture Lr. Pos- } \\
\text { sible herniated } \\
\text { disc }\end{array}$ & $\begin{array}{l}\text { Intercostal } \\
\text { neuralgia. } \\
\text { Multiple rib } \\
\text { fractures }\end{array}$ & - & - & $\begin{array}{l}\text { Laminectomy. Intradural rhizotomy LI } \\
\text { and L2 bilateral after myelogram normal. } \\
\text { Paraparesis, sensory level, neurogenic } \\
\text { bladder after operative haemorrhage and } \\
\text { cord retraction. }\end{array}$ \\
\hline 52 & $M$ & + & & - & + & $\begin{array}{l}\text { Chronic pain due } \\
\text { to disc disease } \\
\text { neuritis } \\
\text { arachnoiditis }\end{array}$ & $\begin{array}{l}\text { Recurrent or } \\
\text { residual disc } \\
\text { herniation and } \\
\text { scar L4-5 }\end{array}$ & + & + & $\begin{array}{l}\text { Intrathecal hypertonic saline. Paraparesis } \\
\text { with sensory level and neurogenic bladder. }\end{array}$ \\
\hline
\end{tabular}


TABLE II

Gamut of general deviations

\begin{tabular}{lc}
\hline & Instances \\
\hline Failure to X-ray appropriate level of spine & I \\
Reliance on discography & 2 \\
Delay in operation in presence of myelographic block & 6 \\
Aspiration of pantopaque below myelographic block & 6 \\
Repeated myelograms in presence of arachnoiditis & I \\
Lesion overlooked because inappropriate low insertion myelogram needle & \\
obscured disease level & I \\
Operation wrong level for artefact caused by needle & I \\
Operation where no myelographic abnormality present consistent with & \\
symptoms or findings & 5 \\
Operation at multiple levels not justified by myelogram & 5 \\
Operation wrong level & 5 \\
Flexion of neck on operating table & 3 \\
Uncorrected and unanticipated hypotension in sitting position & 3 \\
No monitor for air embolus & I \\
Premature, inappropriate, or unnecessarily radical procedure for pain relief & 5 \\
Unnecessarily radical cervical laminectomy & 7 \\
Unnecessarily radical lumbar laminectomy & 4 \\
Too limited laminectomy in presence of narrow canal & 3 \\
Too limited laminectomy for extent of disease & 3 \\
Inappropriate laminectomy for anterior lesion & I \\
Foramenotomies before laminectomy in presence of narrowed canal & I \\
Power drill for foramenotomies or ridge in confined spaces & 2 \\
Unnecessary deliberate dural openings & 5 \\
Dural tears & 2 \\
Opening dura in presence of obvious infection & I \\
Opening dura in presence of obvious metastatic extradural malignancy & 2 \\
Dura left open unnecessarily & I \\
Inappropriate cord incision & 2 \\
Rnappropriate cord biopsies & 2 \\
Torn roots & I \\
Excessive retraction roots & 2 \\
Failure to preserve blood supply roots & 9 \\
Excessive coagulation & 2 \\
Inadequate control of haemorrhage & 2 \\
Foreign body left in wound & 2 \\
Intradural injection of steroids & 2 \\
\hline & I \\
\hline
\end{tabular}

the safeguards insisted upon by the surgeon who introduced the method (Cloward, 1958) or with subsequent updates on the operation.

In general, the adequacy of a surgical procedure could be judged only by its description, its timing, and its appropriateness. Although in some instances questions might reasonably be raised about operative reports which were dictated long after surgery, and after the complication was recognised, or about operations which were unusually protracted, for the purpose of this study such considerations were set aside and the operation was judged by the content of the report, provided that there were no other obvious evidences of unacceptable surgical deviation (e.g. operative or post-operative radiographs). 
PAPERS READ AT THE ANNUAL SCIENTIFIC MEETING, I978

TABLE III

Deviations during Cloward type anterior fusion procedures

\begin{tabular}{lc}
\hline & Instances \\
\hline Multiple unnecessary operative levels & 4 \\
Posterior placement of marker needle in disc space & I \\
Initial drilling to remove entire disc & 3 \\
No measurement of depth of drill site & 4 \\
Power drill for disc space in Cloward procedure & I \\
Excision posterior longitudinal ligament & 5 \\
Interference with blood supply & I \\
Omission necessary instruments & 4 \\
No use of spreaders to inpact graft & 4 \\
Free hand inaccurate shaping of dowel graft & I \\
Manual cervical traction to impact graft by anaesthetist & 3 \\
Graft in spinal canal & 2 \\
Post-operative re-exploration limited to anterior wound & 2 \\
\hline
\end{tabular}

Post-operative care. Treatment during the period immediately following a procedure was below standard in 44 of $5 \mathrm{I}$ circumstances ( 86 per cent). Because of delayed post-operative examination the occurrence of acute complication was long overlooked in eight instances. On I4 occasions there was a failure to determine the cause of it or to reoperate for post-operative paralysis.

It should also be noted that as far as could be determined by these records patients were not questioned at length regarding their symptoms, physical examinations were generally inadequate, and progress notes were infrequently and poorly recorded. There was also limited discussion with these patients of either the rationale for their treatment or the alternatives which might be considered along with the relative advantages and the risks.

\section{Discussion}

Commonly, rumour has it in medical circles that there exist surgical hands, so gilded by skill, that whatever may be the inappropriateness of a particular operation, results which are excellent can nevertheless be anticipated. This writer is certain that such surgeons are surely no phantoms of our imagination, and that they do carry off many operations quite well and unnoticed, in spite of surveillance by good as well as by lax hospital auditing committees. Such doctors are also likely to be surgeons who are canny enough to estimate the tolerance that a patient may have for a particular procedure. The numbers of such doctors, however, cannot be determined on the basis of bad results, and necessarily the search for them must proceed along other lines than these in order to establish some sort of profile by which they can be identified. This is appropriate because they do drain away an unreasonable and substantial share of our health resources.

My interest, however, is with the profile of the surgeon who would appear not to know how to operate. For of paramount concern to us should be the startling fact, borne out by this study, that such a doctor is also not apt to know how to reach a diagnosis or whether, when, or even where to operate. Also, having operated, he usually does not know how to take care of the patient he has injured. These records reflect many more of his inadequacies. The fact that the surgeons 
who are responsible for this poor performance are almost invariably well-trained, board certified specialists, adds necessarily to our amazement.

Under the circumstances the question should certainly not go begging of the intent of these doctors. If it was proper, then did those of them who would appear to know so little ever know much more? If it was not proper, then what motivated an indifference to the fate of their patients? What were the lures, distractions and conflicts which overrode the basic professional ethic to do no harm?

On the basis of this study it can be asserted that these bad results (Table IV) relate to a general failure, to a veritable collapse, of the surgeon as a clinical practitioner of his specialty. They were not 'adverse results' all to be taken as the inevitable outcome of patient care under certain clinical circumstances. They were not 'failed back syndromes'. Nor were they the consequence of 'anatomical anomalies' or 'the inexorable progression' of some disease. Surgeons offer such empty excuses.

It was not overlooked that undesirable results might conceivably relate only to a particular inadequate surgical method or a solitary mishap. There was not a single patient, however, in this study whose clinical evaluation or care was only defective surgically or from a narrow standpoint of operative technique. The data was also looked at from several other angles to test any possibility of more restricted areas of clinical failing which could bias the results. For example, whether or not the patient received appropriate conservative treatment did not alter the diagnostic accuracy at all, and affected the surgical adequacy or postoperative care no more than 2-4 per cent. Conversely, patients receiving substandard surgical treatment showed only a 2 per cent variance from the overall percentile in their likelihood of having received adequate conservative management, and those whose operative care met the standard, paradoxically fared even less well in this regard. These patients, who came to these surgeons for help, were likely to receive treatment across the board which was randomly but consistently poor in any category which one ordinarily examines.

Surgeons argue on occasion that adequate conservative or medical treatment can be presumed on patient referral. It would seem to this writer that such an attitude invites the most perilous oversights. The surgeon, furthermore, must

TABLE IV

Major adverse results

\begin{tabular}{lr} 
Death & 3 \\
Tetraplegia & 7 \\
Tetraparesis & I \\
Triparesis & 4 \\
Paraplegia & I4 \\
Paraparesis & 7 \\
Upper biplegia & I \\
Hemiparesis & I \\
Upper monoparesis & I \\
Causalgia & 2 \\
Occlusion vertebral artery & I \\
Cauda equina syndrome & 4 \\
Root injury pain and deficit & 6 \\
Severe pain & 2 \\
Arachnoiditis & 3 \\
\hline
\end{tabular}


surely need to know better than any other therapist what constitutes appropriate conservative treatment. This is really the first responsibility of a surgeon for it is in line with his obligation to know when not to operate and to know when surgery is not necessary. He is not, after all, a mere technical resource. He must know what can be achieved through non-invasive methods. If by nothing else, by that commitment to do no harm, he is so obligated. The author has previously identified this commonplace failure to employ ordinarily effective conservative management in a study which affected more than 15,000 patients who were injected and often injured by the drug chymopapain (Sussman, I975).

The methods of this study came to be as a matter of chance curiosity concerning the issue of adverse surgical results in a few cases where an evaluation was solicited. The current growth of controversy over these matters added to the writer's interest. Further involvement came as an act of conscience for few doctors would speak for these patients. Other neurosurgeons, some even outraged but still unwilling to say what is said here, did suggest, however, the referral of these records to me for evaluation. What evolved is what would ordinarily be expected from an academic neurosurgeon-a formal attempt to be objective. And yet, that effort on my part, usually engendered still a different sort of resentment.

The author now offers this data, as he would offer any other, in the hope that it may contribute to the resolution of a problem which is of scientific as well as social importance. Considering that his primary interests lie in different areas, it becomes unreasonable past a certain point, for so many referrals to be made to him regarding these matters. Let some of those distinguished colleagues set their own local houses in order and meet more appropriately their sense of outrage by shouldering their own local burdens.

\section{SUMMARY}

Fifty-two patients sustained spinal cord, nerve root or combined injury following treatment conducted primarily by neurological and orthopaedic surgeons.

Most patients did not receive the benefit of appropriate conservative treatment. Correct diagnosis was usually not arrived at even though diagnostic studies were performed sufficiently well to permit such diagnosis. Surgical treatment fell below standard in 86 per cent of the cases in which the issue of adequacy was relevant. Post-operative care was also generally inadequate. The surgeons often failed to detect, diagnose, or reoperate promptly and properly for post-operative paralysis.

These adverse results were not related to isolated surgical mishaps, to natural course of the disease, or to the inevitable result of treatment. They stem from a general failure of the surgeon to meet his reponsibilities as a practitioner. There are both medical and social implications of this finding.

\section{RÉSUMÉ}

Cinquante-deux patients ont subi une corde spinale, la racine des nerfs ou bien une mélange d'injurie suivant traitement conduit d'avance par le neuro et l'orthopédic chirurgiens.

La majorité des malades n'ont pas eu le bénéfice de traitement approprié. Le correcte diagnostic était d'habitude absent bien que l'étude était faite du diagnostic et bien suffisamment exécutée pour permettre ce diagnostic. Traitement chirurgical tomba sous le standard dans $86 \%$ des cas desquels l'issue de suffisance était applicable. Le soin post-opératoire était aussi généralement insuffisant. Les chirurgiens souvent ne purent détecter, diagnosent ou réopérer promptement et proprement pour paralytic post-opératoire. 
Les résultats ci-dessus n'étaient pas associés seulement aux accidents chirurgicals durant la maladie ou de l'inévitable résultat du traitement. Ils viennent d'échec général du chirurgien de reconnaître ses responsabilités comme un médecin. Voilà deux importances sociaux et médicaux de ces conclusions.

\section{ZUSAMMENFASSUNG}

52 Patienten erlitten Rueckenmark oder Nervenwurzel Verletzungen nach Behandlung von Neurochirurgen oder Orthopaeden.

Die meisten dieser Patienten erhielten nicht die angebrachte konservative Behandlung. Die korrekte Diagnose wurde oft verfehlt obwohl die verfuegbaren diagnostischen Studien die richtige Diagnosis erlaubt haetten. Die chirurgische Behandlung war substandard in $86 \%$. Postoperative Behandlung war ebenfalls im allgemeinen unzulaenglich. Postoperative Paralysis wurde haeufig nicht diagnostiziert oder behandelt.

Die bedauerlichen Resultate waren nicht isolierte chirurgische Kunstfehler oder unvermeidbare Ergebnise der Behandlung. Sie sind zurueckzufuehren auf ein weitverbreitetes Versagen der Chirugen in der Behandlung von Rueckenmerksverletzungen.

\section{REFERENCES}

Cloward, R. B. (1958). The anterior approach for removal of ruptured cervical discs. f. Neurosurg., 15, 602-617.

Horwitz, N. H. \& Rizzoli, H. V. (1967). Post-operative complications in neurosurgical practise. Williams and Wilkins, Baltimore.

RovitT, R. L. (1976). American Association of Neurological Surgeons. Report of Professional Liability Committee, San Francisco.

SussmaN, B. J. (1975). Inadequacies and hazards of chymopapain injections as treatment for intervertebral disc disease. F. Neurosurg., 42, 389-396. 